\title{
Proposta de um Servidor de Dados em Pesquisa Clínica Baseado no Padrão HL7 FHIR de Interoperabilidade
}

\author{
Igor O. Vieira ${ }^{1}$, Jefferson L. B. Marques ${ }^{1}$ \\ ${ }^{1}$ Instituto de Engenharia Biomédica - Universidade Federal de Santa Catarina (UFSC)
}

\begin{abstract}
This paper presents an implementable FHIR server model for health research data, complementing the premise that research should not be considered an isolated activity, but conducted in interoperable structures for operational efficiency and effectiveness. The results obtained demonstrate that it is possible to overcome the great challenge in comparing data from different sources, ensuring that they are sets corresponding to each other.
\end{abstract}

Resumo. Este trabalho apresenta um modelo implementável de servidor FHIR para dados de pesquisa em saúde, complementando a premissa de que pesquisa não deve mais ser considerada uma atividade isolada e sim conduzida em estruturas interoperáveis para eficiência e eficácia operacional. Os resultados adquiridos demonstram que é possivel assim superar o grande desafio na comparação de dados de diferentes fontes, garantindo que conjuntos sejam correspondentes entre si.

\section{Introdução}

A pesquisa na saúde desempenha um papel fundamental no avanço médico e na melhoria dos resultados clínicos. Além disso, tem se tornado claro que a agregação e mineração de dados de saúde de múltiplas fontes são papéis importantes na medicina de precisão. Esses dados precisam ser compartilhados e comparados entre si, o quê apoia o conhecimento baseado em evidências e reduz o custo da realização de estudos [Leroux et al. 2017]. Porém, o grande volume de informação sendo manipulado apresenta uma grande deficiência na padronização do mesmo. Órgãos de pesquisa possuem necessidades singulares e manejam dados heterogêneos em relação a sistemas externos. São necessárias adaptações, que são longas, dispendiosas e complexas, além de qualificação de pessoal para tal. Essas adaptações tornam essas informações aptas para sistemas distintos as utilizarem [Benson and Grieve 2021].

A interoperabilidade surgiu como o conceito necessário para a implementação em sistemas computacionais distintos e como uma importante solução para tais problemáticas. Nesse âmbito, sistemas da área seriam capazes de comunicarem entre si de forma homogênea e transparente, apesar das diferenças de linguagem, interface e plataforma de execução [Braunstein 2018]. Para tal, há a necessidade de se publicar padrões de interoperabilidade abertos e acessíveis aos interessados.

O Fast Healthcare Interoperability Resources (FHIR), um padrão desenvolvido pela Health Level Seven (HL7), tem sido o mais amplamente adotado pela comunidade da saúde e mostra-se ser o candidato ideal para o desafio da interoperabilidade na pesquisa clínica. Com isso, esse trabalho propõe a implementação de um servidor de dados de pesquisa clínica utilizando o HL7 FHIR, que é considerado um padrão emergente e um grande candidato como ferramenta para padronização de dados de pesquisa em saúde [Health Level Seven International 2021]. 


\section{Fundamentação Teórica}

O FHIR é um padrão de comunicação lançado em 2011 pela HL7, uma organização internacional credenciada para a criação de padrões para intercâmbio, integração, compartilhamento e requisição de informações eletrônicas de saúde. Esse novo modelo aproveita dos melhores padrões $w e b$, com foco na implementabilidade e na proposta de facilitar a troca de informações. O FHIR foi fundado a partir de um conjunto de componentes chamados de Resources. Eles são as bases para a solução de problemas clínicos e administrativos, com menos complexidade e custos [Health Level Seven International 2021].

O FHIR possui um conceito com quatro paradigmas: interface REST, documentos, mensagens e serviços. Por uma interface RESTful (Representational State Transfer) e API (Application Programming Interface) ocorre o intercâmbio de documentos, pelo envio e recebimento de mensagens que expõem e invocam serviços. Ou seja, a arquitetura RESTful API atua na manipulação dos Recursos com um conjunto de interações (e.g., Create, Read, Update, Delete), que já são amplamente utilizadas na web [Maxhelaku and Kika 2019]. Os Resources são representados em JSON (JavaScript Object Notation) ou XML (Extensible Markup Language). Ambos são formatos leves de troca de dados, fáceis de serem gerados e analisados por máquinas e também compreensíveis para humanos [Solbrig et al. 2017].

Os blocos essenciais de intercâmbio, os Resources, são as representações das instâncias de algum tipo de elemento de saúde, seja dados demográficos, administrativos, observações clínicas, dentre diversos outros. Atualmente estão disponibilizados cerca de 140 Recursos na especificação e todos com características em comum. Ambos possuem um URL (Uniform Resource Locator) que identifique o Recurso, metadados, narrativa XHTML (Extensible Hypertext Markup Language) legível para humanos, além de poderem referenciar uns aos outros [Hawig et al. 2019]. Por fim, todo Resource exige um conjunto de elementos de dados que são previamente definidos para cada tipo, como apresentado no exemplo de um Recurso Patient na Figura 1. Esses elementos possuem tipos de dados primitivos (e.g., boolean, integer, string) ou definidos pelo padrão (e.g., Quantity, Range, CodeableConcept), mas todos detalhados na especificação [Saripalle 2019].

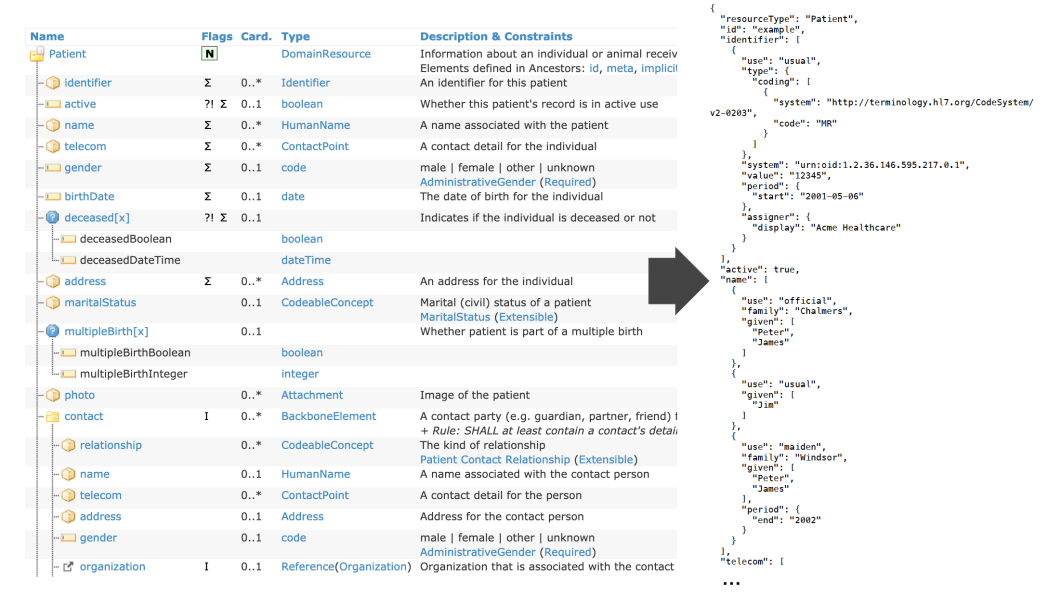

Figura 1. Estrutura de um Resource Patient (esquerda) e de um exemplo em JSON (direita) 


\section{Materiais e Métodos}

A implementação do servidor proposto agrega os seguintes módulos: o mapeamento dos dados para o padrão FHIR, a anonimização de dados pessoais e/ou sensíveis, o banco de dados e a interface RESTful API protegida.

Para a utilização dos dados gerados por uma pesquisa é necessário um estudo preliminar dos mesmos, pois grande parte deles se encontram em formatos distintos dos aceitos pelo FHIR. Para estudo de caso, foram utilizados relatórios com extensão TXT, JSON e CSV (Comma-separeted Values), todos facilmente mapeados para o JSON padrão do FHIR com o Python. Para projetos que queiram se agregar ao servidor, há uma grande facilidade em desenvolver relatórios de saída em XML ou JSON, evitando futuros mapeadores. Além disso, foi possível anonimizar os dados sensíveis e pessoais de pacientes participantes das pesquisas, priorizando a proteção e privacidade dos dados.

O servidor foi desenvolvido a partir do HAPI-FHIR, um projeto aberto de servidor JPA (Java Persistence API). Optou-se por um banco de dados PostgreSQL para armazenamento dos recursos, histórico, indexações, tags e demais dados. O servidor fornece assim uma interface com serviços RESTful, aceitando operações HTTP tais como GET, POST, PUT, DELETE e protegidos por um gerenciador de autenticações. A implementação ainda oferece uma interface que pode ser utilizada em navegador, para melhor compreensão e visualização dos dados (Recursos) e serviços (API), como observado na Figura 2 [Smile CDR 2021].

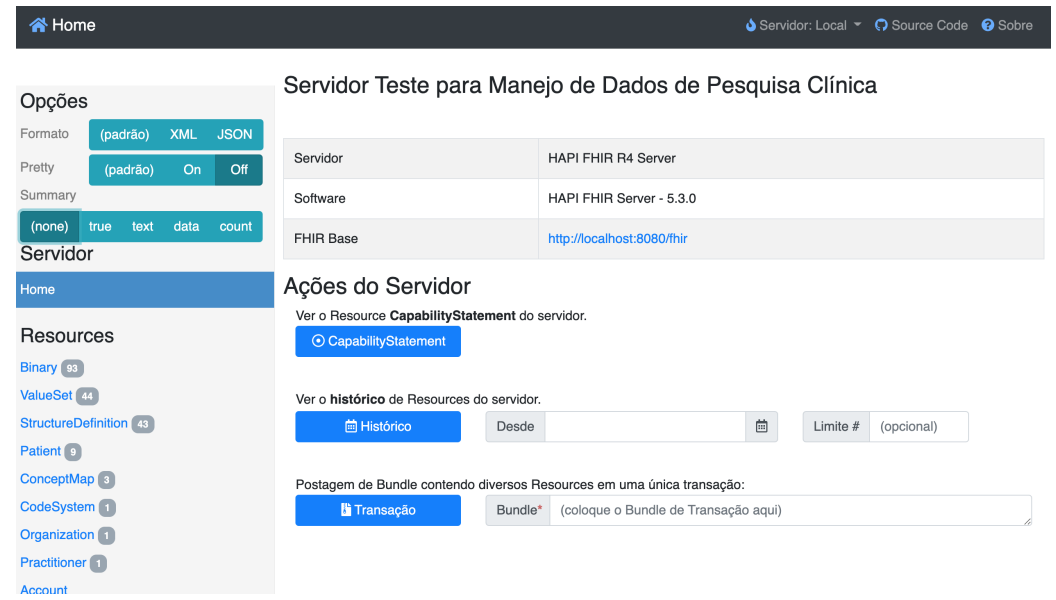

Figura 2. Interface Gráfica do Servidor

\section{Discussão}

O FHIR tem se mostrado a ponte para superação das deficiências técnicas dos padrões de saúde existentes, favorecido pelo crescente poder da computação ubíqua e dos serviços web [Saripalle 2019]. Assim sendo, o servidor mostrou ser bastante eficaz no armazenamento e requisição de dados de pesquisa de um banco de dados local e externo, por meio da interface RESTful. Também foi possível realizar a validação de Resources enviados ao banco de dados para verificação do modelo de dados (conformante ao padrão FHIR) e das terminologias utilizadas pelas informações clínicas, ou seja, os Recursos são aceitos apenas quando validados pelo sistema. 
Para o estudo de caso de uso local e institucional, o servidor requisitou infraestrutura computacional básica (para servidor local ou computação em nuvem), com baixos custos e de fácil manutenção periódica. Além do mais, a proposta torna possível ainda agregar e estender os serviços do servidor com centenas de aplicações de código livre disponibilizados na internet para a implementação em FHIR, seja para mapear Recursos, desenvolver aplicativos, dentre outros. Portanto, esse padrão possui extensa possibilidade de aplicação, seja mobile, comunicação em nuvem e comunicação de servidores, com grandes atributos em segurança e velocidade [El-Sappagh et al. 2019].

\section{Considerações Finais}

Este trabalho apresentou um modelo implementável de servidor FHIR para dados de pesquisa em saúde, complementando a premissa de que pesquisa clínica não deve mais ser considerada uma atividade isolada e sim conduzida em estrutura de redes com troca contínua de dados para eficiência e eficácia operacional. Os resultados adquiridos demonstram que é possível assim superar o grande desafio na comparação de dados e resultados de diferentes fontes, ou seja, garantir que os conjuntos sejam classificados e correspondentes entre si.

\section{Referências}

Benson, T. and Grieve, G. (2021). Principles of Health Interoperability. Health Information Technology Standards. Springer International Publishing, Cham, 4 edition.

Braunstein, M. L. (2018). Health Informatics on FHIR: How HL7's New API is Transforming Healthcare. Springer International Publishing, Cham.

El-Sappagh, S., Ali, F., Hendawi, A., Jang, J. H., and Kwak, K. S. (2019). A mobile health monitoring-and-treatment system based on integration of the SSN sensor ontology and the HL7 FHIR standard. BMC Medical Informatics and Decision Making, 19(1):1-36.

Hawig, D., Zhou, C., Fuhrhop, S., Fialho, A. S., and Ramachandran, N. (2019). Designing a Distributed Ledger Technology System for Interoperable and General Data Protection Regulation-Compliant Health Data Exchange: A Use Case in Blood Glucose Data. Journal of Medical Internet Research, 21(6).

Health Level Seven International (2021). HL7 International Website. http://hl7.org/fhir/.

Leroux, H., Metke-Jimenez, A., and Lawley, M. J. (2017). Towards achieving semantic interoperability of clinical study data with FHIR. Journal of Biomedical Semantics, $8(1): 41$.

Maxhelaku, S. and Kika, A. (2019). Improving Interoperability in Healthcare Using HL7 FHIR. International Academic Conference, pages 35-42.

Saripalle, R. K. (2019). Fast Health Interoperability Resources (FHIR): Current Status in the Healthcare System. International Journal of E-Health and Medical Communications, 10(1):76-93.

Smile CDR (2021). HAPI FHIR: The Open Source FHIR API for Java. https://hapifhir.io/.

Solbrig, H. R., Prud'hommeaux, E., Grieve, G., McKenzie, L., Mandel, J. C., Sharma, D. K., and Jiang, G. (2017). Modeling and Validating HL7 FHIR Profiles Using Semantic Web Shape Expressions (ShEx). Journal of Biomedical Informatics, 67:90100 . 